\title{
Anal Sexual Practices among Undergraduate Students
}

\author{
Cristinia Molinares, Irina Kolobova, and David Knox \\ East Carolina University
}

\section{Introduction}

Approximately $30 \%$ of women and $35 \%$ of men report having engaged in heterosexual anal intercourse (HAI) in the past year (Hess, Reynolds, \& Fisher, 2014). Other researchers have reported that between $1 \%$ and $40 \%$ of women and $8 \%$ and $43 \%$ of men have engaged in HAI at least once in their lifetime (Fahs, Swank, \& Clevenger, 2015). The rate of HAI among college students is similar to that of the general population. Halperin (1999) reported that approximately $25 \%$ of students at the University of Maryland had engaged in HAI.

McBride and Fortenberry (2010) reviewed the literature on anal sex and noted that an Internet search for the term "heterosexual anal sex" yielded 790,000 links related to pornographic images and chats, scientific findings, and anal sex health information. The researchers suggested that anal sex is sometimes regarded as the "new" oral sex. A cultural shift in sexual norms may contribute to an increased prevalence of heterosexual anal sex. More women today are engaging in anal intercourse than previously. Data from 1991 and 1992 revealed that only $18-20 \%$ of women had ever engaged in anal sex compared with $28-33 \%$ in 2002, thus demonstrating an increase over a ten-year period (Fahs et al., 2015).

Even though data suggest that more individuals are engaging in HAI, there has only been limited research focusing on the motives for engaging in HAI. Thus far, researchers have identified religion, being in a monogamous/committed relationship and pleasure as motives for engaging in HAI (Fahs et al., 2015; McBride \& Fortenberry, 2010). Some religious individuals engage in anal intercourse as opposed to vaginal intercourse in order to maintain their virginity (Fahs et al., 2015) since they do not consider anal intercourse as "real" sex (e.g., sexual intercourse) (McBride \& Fortenberry, 2010).

Being in a committed relationship is also associated with engaging in anal sex. Trust has been identified as being higher in committed relationships compared to casual relationships. Reynolds, Fisher, and Rogala (2015) found that some of their female respondents felt less at risk for contracting an STI while engaging in unprotected heterosexual anal sex with a committed partner whom they trusted.

Pleasure is also a motivation for anal sex with men reporting more positive HAI than women. Almost sixty percent of heterosexual male participants compared to 13 percent of heterosexual female participants in Fahs et al. (2015) research reported having enjoyed their past anal sex experience. Other researchers have found that some women engage in HAI for their own enjoyment (Reynolds et al., 2015).

The purpose of this study was to move beyond the existing data and examine other motives for heterosexual anal intercourse. While previous research has focused mainly on high 
risk populations such as substance users (Reynolds et al., 2015), the homeless (Hess et al., 2014), and men who have sex with men (Fahs et al., 2015; Hess et al., 2014), this study examined the motives for and interest in anal intercourse among a sample of heterosexual college students. First, we look at two theories which serve as the foundation for understanding anal sex as an increasing phenomenon.

\section{Theoretical Frameworks}

Sexual script theory and sexual liberalism provide the lens for viewing contemporary anal sex practices. Sexual script theory emphasizes that an individual's sexual behavior is influenced by social scripts which reflect a person's gender (Sakaluk, Todd, Milhausen, \& Lachowsky, 2014). Due to the biological differences between men and women, the respective genders are socialized with different beliefs and values (Sakaluk et al., 2014). Traditional sexual scripts are entrenched (Sakaluk et al., 2014) and reflect traditional cultural gender norms (Sanchez, Fetterolf, \& Rudman, 2012). For example, a study on anal sex practices among Puerto Rican university students found that $47 \%$ of the heterosexual men either "always" or "almost always" made the decision about having anal sex (Halperin, 1999). Eighty percent of the female participants reported that $65 \%$ of the time their partner "always" made the decision to have anal sex (Halperin, 1999). These data reflect the cultural norm in Puerto Rico that it is the male's role to initiate and make decisions about sexual intimacy (Sakaluk et al., 2014; Sanchez et al., 2012).

However, the world is changing. Sexual liberalism includes being the "Neoliberal" female, seeking to be free (not tied down to one partner), and sexually autonomous (Bay-Cheng \& Goodkind, 2016). Sexual liberalism also implies that women are becoming more assertive in initiating, enjoying, and not feeling guilty about engaging in sexual behavior. While men may be initiating sex more often, women are both initiating and enjoying it too. We return to this theme in the discussion section.

\section{Method}

A descriptive, cross-sectional design was utilized to collect data from undergraduate women and men about their attitudes and beliefs regarding anal intercourse at a moderately sized southeastern university. Data were collected through a self-administered online survey via Qualtrics (Qualtrics, 2005). The study was approved by the Institutional Review Board.

\section{Sample and Procedure}

Undergraduate students in the third author's sociology courses were invited to participate in the research during the Fall 2015 semester. The students were emailed a link to the online survey and asked to complete the anonymous and voluntary survey. All students who completed the survey were at least 18 years of age.

\section{Measures}

Participants completed a 53-item survey (see Appendix A) developed by the authors that consisted of categorical questions and rating scales about participants' demographic information, sexual attitudes, and behaviors. An example of a question about a participant's sexual attitudes 
was, "On a ten-point scale, to what degree are you open to new sexual experiences?" An example of a question about sexual behavior was, "Have you engaged in anal intercourse?" Participants that reported ever engaging in anal intercourse responded to additional questions about their experiences, beliefs, and satisfaction. An example of a question of this nature was, "What are your feelings about having participated in anal sex?"

\section{Statistical Analysis}

A variety of statistical techniques (univariate, bivariate, and multivariate analyses) were used to analyze the data. Descriptive univariate analyses were used to summarize the data and identify demographic characteristics of the sample. Chi-square and independent sample t-tests were used to compare variables across groups (e.g., those who engaged in anal sex compared to those who had not, male participants compared to female participants). Additional bivariate analysis with only those participants who reported having engaged in anal intercourse was also completed. Statistical significance was set at a $\mathrm{p}$-value of $<0.05$ and pairwise deletions were used for missing cases. All statistical analysis was performed using SPSS software.

\section{Results}

Of the 265 undergraduates who were emailed the link, 189 completed the anonymous/voluntary survey (71\%). Table 1 details the sample's demographic characteristics. Table 1.

Sample Characteristics $(N=189)$

\begin{tabular}{lll}
\hline Frequency (\%) or Mean (SD) & \\
\hline Gender & Male participants & $29(15.3)$ \\
& Female participants & $159(84.1)$ \\
& Transgender participants & $1(.5)$ \\
\hline Race & White & $133(70.4)$ \\
Black & $34(18)$ \\
Hispanic & $8(4.2)$ \\
Asian & $5(2.6)$ \\
Native American & $3(1.6)$ \\
Bi-racial & $6(3.2)$ \\
\hline Sexual orientation & $175(92.6)$ \\
Heterosexual & $6(3.2)$ \\
Homosexual & $5(2.6)$ \\
Bisexual & $2(1.1)$ \\
Pansexual & $1(.5)$ \\
Asexual & $30(15.9)$ \\
Freshman & $80(42.3)$
\end{tabular}




\begin{tabular}{ll} 
Junior & $47(24.9)$ \\
Senior & $29(15.3)$ \\
Graduate student & $3(1.6)$ \\
\hline Grade point average $(\mathrm{N}=188)$ & $6(3.2)$ \\
2.0 or lower & $45(23.9)$ \\
$2.1-2.8$ & $79(42)$ \\
$2.9-3.4$ & $43(22.9)$ \\
$3.5-3.7$ & $15(8)$ \\
3.8 or higher & \\
Mother's highest level of education & $2(1.1)$ \\
Did not finish high school & $35(18.5)$ \\
High school graduate & $37(19.6)$ \\
Some college & $80(19.6)$ \\
College graduate & $30(15.9)$ \\
Master's degree & $5(2.6)$ \\
PhD, MD, or JD & \\
Not dating status & $71(37.6)$ \\
Casually dating & $29(15.3)$ \\
Committed relationship with & \\
one person & $84(44.4)$ \\
Engaged & $3(1.6)$ \\
Married & $2(1.1)$ \\
\hline
\end{tabular}

The majority of the respondents were women (84\%), white (70\% with $18 \%$ black, $4 \%$ Hispanic) and heterosexual (93\%). Over half (58\%) of the sample identified as freshman or sophomores. Due to the small number of non-heterosexual participants $(n=14)$, additional analyses was limited to heterosexual participants $(n=175)$.

Less than half (41.3\%) of the sample reported having engaged in any type of sexual activity and over a third (34.5\%) of the sample reported having engaged in anal intercourse. An interesting observation is that among those who reported never having engaged in any type of sexual activity, 26\% reported having engaged in anal intercourse, suggesting some of the respondents did not view anal sex as "real" sex (e.g., sexual intercourse) or did not understand the question. Despite this observation, their responses were included in the additional analyses.

With regard to sexual values, $9 \%$ of the respondents were absolutist (e.g., virginity till marriage), 32\% were hedonistic (e.g., sex focused on pleasure), and 59\% were relativistic (e.g., sex in a relationship). Twenty percent of the respondents reported having had over 10 previous sexual partners. Additional descriptive results regarding participants' sex values and behaviors are presented in Table 2 . 
Table 2.

Sex values and beliefs of heterosexual participants $(N=175)$

Own sexual attitude

Sex before marriage is wrong

Sex is ok in a relationship

$16(9.1)$

"If it feels good, do it"

$103(58.9)$

Parents' sexual attitudes

Very open about sex

$56(32)$

Household taboo

$31(17.6)$

Hold traditional sexual values 34 (19.3)

Acknowledged but not

discussed

$98(55.7)$

Number of sexual partners $(n=173)$

0

1

$2-5$

$54(30.7)$

6-10

$22(12.5)$

$11-20$

$22(12.5)$

$21-50$

$9(5.1)$

$51-75$

$3(1.7)$

Engaged in anal sex $(\mathrm{n}=171)$

Yes

No

Overall, male participants had a higher degree of openness for sexual experiences (men: $M=7.92, S D=1.863$; women: $M=6.19, S D=2.154 ; t(172)=3.702, p<.001)$, a higher interest in anal sex (men: $M=5.19, S D=2.792$; women: $M=2.45, S D=2.287 ; t(172)=5.241, p<$

.001 ) and a greater openness for anal intercourse (men: $M=6.00, S D=2.993$; women: $M=3.07$, $S D=2.570 ; t(181)=5.069, p<.001)$ compared to women. Female participants reported a higher level of disgust $(M=6.07, S D=3.319)$ than male participants $(M=4.63, S D=3.214 ; t$ $(172)=1.984, p<.05)$ about anal intercourse. Male participants were more likely to have suggested anal sex to their partner (59\%) compared to female participants $\left(16.1 \% ; X^{2}(1)=\right.$ 21.502, $p<0.001)$. Female participants reported a higher level of pressure from their partners to engage in anal intercourse $(M=2.69, S D=2.257)$ as compared to male participants $(M=1.48$, $S D=1.310 ; t(47.294)=3.624, p=.01)$.

Thirty percent of the female and $37 \%$ of the male participants reported that they had experienced anal intercourse. The primary reason reported for engaging in anal sex was "to please the partner" $(32 \%)$, with female participants more likely than male participants to report this as a reason. Other motives included curiosity (27\%) and "to spice things up" (10\%). Less than half of the sample reported that alcohol was involved during their last anal intercourse experience. Less than $10 \%$ of the sample reported that drugs (other than alcohol) were involved during the last anal intercourse experience. There were no gender differences with regard to the presence of alcohol or drugs. 
Other findings revealed that those who had engaged in anal intercourse reported being less religious than those who had not engaged in anal intercourse $\left(X^{2}(4)=10.208, p<.05\right)$. Rates of anal intercourse were lowest among female participants who held absolutist sexual values and highest among those who held hedonist sexual values $\left(X^{2}(2)=8.819, p<.05\right)$. Having engaged in anal intercourse was higher among juniors and seniors compared to freshmen and sophomores $\left(X^{2}(4)=10.992 p<.05\right)$. Participants in committed relationships were more likely to report having engaged in anal intercourse $\left(X^{2}(4)=10.742, p<.05\right)$.

Overall, male participants evaluated anal intercourse higher $(M=7.00, S D=2.530)$ than female participants $(M=4.19, S D=2.924 ; t(56)=2.934, p=.005)$. Sixty-four percent of the men compared to $44 \%$ of the women reported positive feelings about participating in anal intercourse. None of the men and $9 \%$ of the women reported regretting the experience. Male participants were more likely to report that they initiated anal intercourse $(54.5 \%)$ compared to female participants $\left(15.2 \% ; X^{2}(1)=7.799, p<.01\right)$.

Finally, male participants were more likely to report having had an orgasm during the last anal intercourse experience $(72.7 \%)$ compared to female participants $\left(25.5 \% ;\left(X^{2}(1)=8.788, p\right.\right.$ $<.01)$. In a multiple regression model predicting evaluation of anal intercourse that included gender and orgasm, only having an orgasm was significant $(B=-.634, p<.001)$ in predicting a higher evaluation of anal intercourse $(F(2,55)=24.775, p<.001)$.

\section{Discussion}

This study examined the motives for and interest in heterosexual anal intercourse (HAI) among a sample of college students. While research suggests that rates of engaging in HAI are increasing (Hess et al., 2014; Fahs et al., 2015), the literature is sparse regarding the motives for HAI. Findings from this study reflect new insights in regard to anal sex practices among heterosexual undergraduate students. Over a third (34\%) of the sample reported engaging in the behavior, which is more than previously reported for a sample of students at the University of Maryland (Halperin, 1999). The higher rate among college students parallels the change in rates among the non-college population (Hess et al., 2014; Fahs et al., 2015).

Participants endorsed a variety of reasons for engaging in HAI, including to please their partners, personal curiosity, and to "spice things up". Previous researchers have identified religion, being in a committed relationship and pleasure as motives (Fahs et al., 2015; McBride $\&$ Fortenberry, 2010). While overall, in this study, men evaluated HAI higher than women, 44\% of women reported enjoyment with less than ten percent (9\%) reporting any regret. A quarter $(25.5 \%)$ of the female participants who had engaged in HAI reported having experienced orgasm and it is these females who reported greater enjoyment in anal sex. Furthermore, gender alone did not predict a positive evaluation of anal sex but rather, having an orgasm. This finding suggests that pleasure may be an important aspect of how individuals experience HAI and may serve as a motive for some individuals. This finding supports previous findings suggesting that some women engage in HAI for their own enjoyment (Reynolds et al., 2015).

\section{Implications}


This study provides evidence that undergraduate students are engaging in HAI at greater rates than previously reported (Halperin et al., 1999) and that women may be engaging in HAI for personal reasons such as curiosity and pleasure. These findings may be particularly useful to healthcare educators, healthcare providers, and researchers who work with undergraduate students.

There are three implications from these findings to consider. First, only a small portion of the sample reported having absolutist views on sexual activity and $41.3 \%$ of the sample had previously engaged in sexual activity. More than a third of the sample had engaged in heterosexual anal intercourse. These findings emphasize that undergraduate students are engaging in sexual activities and even more are likely discussing topics related to sexual activity. If anal sex is a "taboo topic," this was not the case in our sample. While men were more likely to suggest HAI, women participated in and enjoyed HAI which supports the need for both men and women to receive comprehensive and timely information about healthy sexual activity from educators and healthcare providers. Information about safe sex practices should be geared towards empowering men and women to be responsible with their bodies.

Another implication from the findings is that gender roles are changing. One in three female participants reported experiencing anal sex, and of these, four in ten reported enjoying the experience. Less than ten percent of the women reported regretting the experience. Women of today who are sexually assertive initiate and communicate about the sex they desire ("I feel comfortable telling my partner what I want him to do"), refuse unwanted sex ("If I don't want to do something, I tell him"), and communicate with their partners about sexual history and risk ("I ask my partner if he has practiced safe sex with other partners") (Loshek \& Terrell. 2015). Women are shifting from traditional sexual scripts and embracing sexual liberalism. Future research to understand this shift may be beneficial for empowering women to continue to ask for what they desire and to not feel pressured to engage in unwanted sexual activities.

The final implication of this research is in reference to the high rate of orgasm during HAI experienced by female participants. While women in general report experiencing predictable orgasm three to ten percent of the time via vaginal intercourse (Mintz, 2017), over a quarter of the female participants in this sample reported an orgasm during their most recent HAI experience. Experiencing an orgasm, not gender, was a significant factor in predicting a higher evaluation of HAI. This finding emphasizes the role of pleasure in women's sexual experiences and highlights the need for more research about women's sexual pleasure. Understanding the role of pleasure in women's decision making process is important to healthcare educators and providers who are working with women on building positive sexual identities.

\section{Limitations}

While this study provides valuable insight into motives for HAI for undergraduate students, there are several limitations. First, the convenience sample of 189 undergraduate students is hardly representative of the almost 20 million undergraduate students throughout the United States. This sample was collected from a public university in the southeastern portion of the United States and may not be representative of other regions of the country. Second, the data 
are quantitative with no qualitative interviews to provide insights on the raw statistics. Subsequent research on anal sex might include interviews about this private subject to understand how pleasure, social scripts, and expectations influence the decisions men and women make with regard to HAI. Third, the final sample only included heterosexual undergraduate students. Individuals who engage in same sex anal intercourse may have different motives for engaging in it and experiences with it. Additional research that compares these different populations would be beneficial for building an understanding of anal intercourse experiences.

\section{Conclusion}

This cross sectional study provides original findings regarding HAI experiences among a sample of undergraduate students at a southeastern university. Rates of HAI are increasing among undergraduate students and both men and women are enjoying the experience. As healthcare educators, providers, and researchers aim to meet the needs of sexually active undergraduate students, educating and empowering them about positive sexuality is likely to have better outcomes for all involved.

Bay-Cheng, L. \& Goodkind, S. (2016). Sex and the single (Neoliberal) girl: Perspectives on being single among socioeconomically diverse young women. Sex Roles, 74 (5/6), 181-194.

Fahs, B., Swank, E., \& Clevenger, L. (2015). Troubling anal sex: Gender, power, and sexual compliance in heterosexual experiences of anal intercourse. Gender Issues, 32, 19-38. doi:10.1007/s12147-0149129-7

Halperin, D. T. (1999). Heterosexual anal intercourse: Prevalence, cultural factors, and HIV infection and other health risks, part I. Aids Patient Care and STDs, 13(12), 717730. doi:10.1089/apc.1999.13.717

Hess, K. L., Reynolds, G. L., Fisher, D.G. (2014). Heterosexual anal intercourse among men in Long Beach, California. Journal of Sex Research, 51(8), 874-881. doi:10.1080/00224499.2013.809512

Loshek, E. \& Terrell, H. K. (2015). The development of the sexual assertiveness questionnaire (SAQ): A comprehensive measure of sexual assertiveness for women. The Journal of Sex Research, 52 (9), 10171027. doi:10.1080/00224499.2014.944970

Journal of Positive Sexuality, Vol. 3, March 2017

\section{References}

McBride, K. M. \& Fortenberry, J. D. (2010). Heterosexual anal sexuality and anal sex behaviors: A review. Journal of Sex Research, 47(2-3), 123-136. doi: $10.1080 / 00224490903402538$

Mintz, L. (2017). Becoming Cliterate: Why Orgasm Equality Matters. New York: HarperOne

Qualtrics (2005). Qualtrics (Version 5.15). Provo, Utah, USA

Reynolds, G. L., Fisher, D. G., \& Rogala, B. (2015). Why women engage in anal intercourse: Results from a qualitative study. Archives of Sexual Behavior, 44, 963-995. doi:10.1007/s10508-014-0367-2

Sakaluk, J. K., Todd, L. M., Milhausen, R. \& Lachowsky, N. J. (2014). Dominant heterosexual sexual scripts in emerging adulthood: Conceptualization and measurement. Journal of Sex Research, 51(5), 516-531. doi:10.1080/00224499.2012.745473

Sanchez, D. T., Fetterolf, J. C., \& Rudman, L. A. (2012). Eroticizing inequality in the United States: The consequences and determinants of traditional gender role adherence in intimate relationships. Journal of Sex Research, 49(2-3), 168-183. doi:10.1080/00224499.2011.653699 


\title{
Appendix A: Survey on Anal Sex Practices among Undergraduate Students
}

\author{
Cristinia Molinares, Irina Kolobova, and David Knox \\ East Carolina University
}

\section{Survey on Anal Sex}

You are invited to participate in a research study titled "Anal Sex: Meanings and Negotiation in Relationships" conducted by David Knox, a sociology faculty member at East Carolina University, Cristinia Molinares (undergraduate senior majoring in sociology) and Irina Kolobova (doctoral candidate in medical family therapy). The goal of the research is to survey 400 individuals enrolled in courses taught by Dr. Knox. The survey will take approximately 15 minutes to complete. The survey is anonymous and confidential. No data will be collected which will identify you. Your participation in the research is voluntary. You may choose not to answer any or all questions, and you may stop at any time. There is no penalty for not taking part in this research study. Please call David Knox at 2527563562 for any research related questions or the Office of Research Integrity \& Compliance (ORIC) at 252-744-2914 for questions about your rights as a research participant.

There is no "capturing of your email or IP address" when you submit this questionnaire. In addition, no identifying code will be attached to any response. Finally, the questionnaire is to be completed by only by individuals age 18 or above. If you are underage, you have received this questionnaire in error; please disregard. The survey can be found at **insert link here**

\section{Background/Demographics}

1. Sex
( ) Male
( ) Female
( ) Transgender

2. I regard myself as:
( ) White
( ) Black
( ) Hispanic
( ) Asian
( ) Native American
( ) Biracial
( ) Other

3. My class standing in college is:
( ) Freshman
( ) Sophomore
( ) Junior
( ) Senior
( ) Graduate Student

4. I define my sexual orientation as:
( ) heterosexual
( ) homosexual
( ) bisexual
( ) transgender
( ) other

5. I consider myself a religious/spiritual person 

( ) Strongly disagree
( ) Somewhat Disagree
( ) Neither disagree nor agree
( ) Somewhat agree
( ) Strongly agree

6. My Grade Point Average (GPA) is:
( ) 2.0 or lower
( ) $2.1-2.8$
( ) $2.9-3.4$
( ) $3.5-3.7$
( ) 3.8 or higher

7. On average, I consume alcohol:
( ) Once per day
( ) Between 3-5 nights a week
( ) Once a month
( ) Several times a year
( ) Almost never
( ) I have never consumed alcohol

8. What is your mothers' highest level of education?
( ) she did not finish high school
( ) high school graduate
( ) some college but did not graduate
( ) college graduate
( ) Master's degree
( ) Ph.D., MD, or degree in law

9. How would you describe your parents' sexual attitudes?
( ) They are very open about sex
( ) Discussion of sex is a household taboo
( ) They hold traditional sexual values (e.g. virginity at marriage)
( ) Sex is acknowledged, but not spoken of often

10. Have you experienced any sexual activities (kissing, touching, fondling, intercourse, oral or anal sex) with someone of the same sex?
( ) yes
( ) no

11. Approximately how many different partners have you had sexual intercourse with?

( ) I have never had sexual intercourse

( ) 1

( ) $2-5$

( ) $6-10$ 

( ) $11-20$
( ) $21-50$
( ) $51-75$
( ) $76-100$
( ) More than 100

12. What is your current relationship status?

( ) not dating and not involved with anyone

( ) casually dating different people

( ) emotionally involved with one person in a committed relationship

( ) Engaged

( ) Married

( ) Divorced

( ) Legally Separated

13. How would you describe the average number of weekly sexual encounters with your partner?

( ) not sexually active with my partner

( ) 1-3 times a week

( ) 3-5 times a week

( ) at least once a day

( ) several times a day

14. What is your level of sexual satisfaction with your partner?

Not Satisfied

Very

Satisfied

1

2

3

4

5

6

7

8

9

10

\section{Sexual Values and Behavior}

15. The sexual value which best describes me is:

( ) absolutism- intercourse before marriage is wrong

( ) relativism- if you are in a loving relationship, intercourse is ok even if not married

( ) hedonism- "if it feels good, do it"- being in love or being married doesn't matter

16. On a ten point scale, to what degree are you open to new sexual experiences?

Not open

1

2

3

4

5

$6 \quad 7$

8

Very open

17. Have you ever watched a pornographic scene involving anal sex?

( ) yes

( ) no 
18. If you have ever watched a pornographic scene involving anal sex, what were the sexes of the participants?
( ) male-male
( ) male- female
( ) female-female
( ) I have never witnessed a pornographic scene of anal sex

19. Has any romantic partner ever suggested that you try anal sex?
( ) yes
( ) no

20. Have you ever suggested anal sex to a romantic partner?
( ) yes
( ) no

21. Given the opportunity, what is your level of interest in having anal sex?

Not interested

Very interested
1
2
3
4
5
$6 \quad 7$
8
9
10

22. On a ten point scale, to what degree are you open to having anal sex?

Never

I am ready now

$\begin{array}{llllllllll}1 & 2 & 3 & 4 & 5 & 6 & 7 & 8 & 9 & 10\end{array}$

23. On a ten point scale, to what degree do you find the idea of participating in anal sex disgusting?

Not at all

1

2

3

4

5

6

7

8

Extremely

24. How many times were you asked by your partner to have anal sex before it happened?

( ) less than 5 times

( ) between 5 and 10 times

( ) we immediately agreed

( ) more than 10 times

( ) my partner never asked me to have anal sex

( ) I have never had anal sex

25. On a ten point scale, how much pressure did you feel from your partner to have anal sex? No pressure 2 3 4 $5 \quad 6$ 7 Extreme Pressure

26. On a ten point scale, how much pressure did you put on your partner to have anal sex? 
No pressure

(1)

2

4

5

6

7

8

Extreme Pressure

$9 \quad 10$

27. Have you engaged in anal intercourse?
( ) yes
( ) no

28. In how many different relationships have you had anal sex?
( ) none
( ) one
( ) two
( ) three
( ) four
( ) five or more

If you have not engaged in anal sex, you have finished the survey. If you have engaged in anal sex, complete the remaining questions. If you have had more than one relationship in which anal sex was included, refer to the last relationship.

29. Describe the nature of your overall anal sex experience in the most recent relationship that has included anal sex.
( ) one time occurrence
( ) occasional occurrence (less than five times) with the same partner
( ) occasional occurrence (less than five times) with different partners
( ) frequent occurrence (more than five times) with the same partner
( ) frequent occurrence (more than five times) with different partners

30. What was your goal in having anal sex?

( ) to spice things up

( ) to live out a fantasy I have had about having anal sex

( ) to please my partner (I knew my partner would like it and I wanted them to enjoy)

( ) curiosity

( ) to explore my sexuality

( ) alcohol or drugs were involved and it just happened

( ) to stop my partner from pressuring me, I gave in

31. Who suggested the idea of having anal sex the first time it occurred?
( ) I did
( ) my partner

32. Did you have an orgasm during your last anal sex experience?
( ) yes
( ) no

33. What is your overall evaluation of having had anal sex? 
Terrible

Wonderful

1 2

3

4

5

6

7

8

9

10

34. Was alcohol involved the last time you had anal sex?
( ) yes
( ) no

35. Were drugs other than alcohol involved during the anal sex?
( ) yes
( ) no

36. When did anal sex with your partner occur?
( ) after vaginal sex
( ) before vaginal sex
( ) there was only anal sex
( ) during foreplay

37. Did you use a condom during your last anal sex experience?
( ) yes
( ) no

38. If anal sex occurred after vaginal intercourse, was the same condom used?
( ) yes
( ) no
( ) I do not know
( ) we did not use a condom
( ) anal sex did not occur after vaginal sex

39. If anal sex occurred before vaginal intercourse, was the same condom used?
( ) yes
( ) no
( ) I do not know
( ) we did not use a condom
( ) anal sex did not occur before vaginal intercourse

40. Did you use any lubrication during your last anal sex experience?
( ) yes
( ) no

The following questions are about your overall experiences with anal sex or play.

41. Have you ever given a "rim" job (tongue on anus)?
( ) yes
( ) no 
42. Have you ever received a "rim" job (tongue on anus)?
( ) yes
( ) no

43. If you have received a "rim" job, how much did you enjoy it?

No enjoyment

Extremely enjoyable

$$
1
$$

4

5

6

7

8

9

10

44. Have you ever given anal stimulation with your fingers?
( ) yes
( ) no

45. Have you ever received anal stimulation via your partner's fingers?
( ) yes
( ) no

46. If you have ever received anal stimulation via your partner's fingers, how much did you enjoy it?

No enjoyment enjoyable

$$
1
$$

2

4

5

$\begin{array}{ll}6 & 7\end{array}$

8

Extremely

47. Have you ever given your partner anal stimulation using a sex toy?
( ) yes
( ) no

48. Have you ever received anal stimulation from your partner using a sex toy?
( ) yes
( ) no

49. If you have ever received anal stimulation from your partner using a sex toy, how much did you enjoy it?

No enjoyment

12

23

4

5

6

7

Extremely enjoyable

50. As a female have you ever "traded" anal sex for cunnilingus- you would let your partner have anal sex in exchange for your partner performing oral sex on you?
( ) yes
( ) no

51. What are your feelings about having participated in anal sex?

( ) I feel ashamed

( ) I am proud

( ) I enjoyed the experience 
( ) I brag about my experience

( ) I keep this a secret

( ) I violated my own values

( ) I regret the experience

( ) I feel dirty

52. What is your level of interest in having another anal sex experience?

Never

Can't

wait

$\begin{array}{lllllllll}1 & 2 & 3 & 4 & 5 & 6 & 7 & 8 & 9\end{array}$

10

53. What was the relationship outcome of having anal sex with your partner?

( ) I have never had anal sex

( ) brought us closer together

( ) created strain in our relationship/more emotionally distant

( ) no effect

( ) we broke up

Thank you for completing the questionnaire. 